\title{
Technique to Generate Variable Keys with Key Variation with Noise Burst Bit for Achieving Perfect Security in Cryptology towards Optimum Data Transfer
}

\author{
Moumita Das ${ }^{1}$, Rajat Subhra Goswami ${ }^{2}$, Manash P Dutta ${ }^{3}$, S. K. Chakraborty ${ }^{4}$ and \\ C. T. Bunia ${ }^{5}$ \\ 1,2,3,4,5 Department of Computer Science \& Engineering \\ National Institute of Technology, Arunachal Pradesh \\ moumita.das23@gmail.com ${ }^{1}$, rajat.nitap@gmail.com ${ }^{2}$, \\ manash.cse@nitap.in ${ }^{3}$, skchakraborty.cse@nitap.in ${ }^{4}$, ctbhunia@vsnl.com ${ }^{5}$
}

\begin{abstract}
To create dynamic keys which change from session to session or data to data, an idea has been found in literature which has been named as Automatic Variable Key. This technique has been proposed to overcome the problem that arises with a static key. In case of static key, the same key has been used for the entire data communication and if anyhow it has been compromised then the basic requirement of cryptography fails. In this manuscript, we have proposed a few dynamic key generation techniques. In these proposed techniques, the variable keys have been generated based on some existing techniques namely, Computing \& Shifting Automatic Variable Key, Alternating \& Shifting Automatic Variable Key, Left Shifting Automatic Variable Key by incorporating noise burst bit. During the performance analysis, it has been observed that the auto generated keys are more random in the proposed techniques. The randomness of successive generated keys have been tested with the help of NIST (National Institute of Standards and Technology), a statistical tool and proved that the generated keys are truly random. Lastly, we have commented that these techniques can be used in real-time applications as they provide more security as compared to other existing mechanisms.
\end{abstract}

Keywords: Randomness, Standard Deviation, Automatic Variable Key, Noise Burst

\section{Introduction}

Information is an asset that has a specific value so; our aim is to keep that information secretly from attackers and to maintain confidentiality of information. However, attaining the security by a single key in today's era becomes an obsolete idea due to the incredible growth of information technology. As per the fundamental research proposed by Shannon [1-2], perfect security can be achieved if the key is made variable from session to session during data transmission in network security. The cryptographic algorithms rely on increasing the key size to enhance the level of security, it will be an effective way, if keys can be made time variant by changing it from session to session in data communication. Vernum [3-4] proposed that if the generated keys would be changed from session to session during data transmission then it would be impossible to break the keys. Bhunia [57] proposed the Automatic Variable Key technique for practical approaches of security in the year 2006. There are many researchers like Goswami [10-17], Banerjee and Dutta et al. [8-9] invented more secure techniques based on Automatic Variable Key (AVK) by increasing the randomness, standard deviation and root mean square among the auto generated key sequences. The aim of our techniques is to secure the key from unauthorized persons and tom hide the secret keys from others and to generate more 
random keys as compared to the existing key generation techniques. The technique of Automatic Variable Key is based on the following procedure:

The initial key $K_{1}$ will be exchanged between sender and receiver in a secret mode. Subsequent keys for different data $D_{i-1}$ will be automatically generated between two parties sender and receiver in secret mode like AES.

One technique was proposed by Goswami in 2013 called Computing \& Shifting Automatic Variable Key (CSAVK) [14]. In this proposed technique, the key is made variable by an agreement that creates new key for each data. Subsequent keys will be generated as per the following equation:

$$
K_{i}=K_{i-1}^{z} \oplus D_{i-1}^{z} \quad \forall \quad \mathrm{i}>0 \ldots \ldots
$$

Where $K_{i-1}^{s}=$ bit wise right shifted key $K_{i-1}$, number of shift will be the number of 1's present in $K_{i-1}$ and $D_{i-1}^{t}=$ bit wise left shifted data $D_{i-1}$, number of shift will be the number of 1's present in $D_{i-1}$.

In Alternating \& Shifting Automatic Variable Key (ASAVK) [15] technique, the key is made variable a procedure that also creates new key for each data.

At first, initial key $K_{1}$ is exchanged between sender and receiver and subsequent key $K_{i}$ $\left(i^{\text {th }}\right.$ stage) is generated by both sender and receiver as follows:

$$
\begin{gathered}
K_{i}=K_{i-1}^{s} \oplus D_{i-1} \quad \forall \mathrm{i}>0 . \ldots \\
K_{i+1}=K_{i} \bigoplus D_{i-1}^{s} \quad \forall \mathrm{i}>0 \ldots .
\end{gathered}
$$

Where $K_{i-1}^{t}=$ block wise shift $K_{i-1}$, the number of shift will be total length of $K_{i-1}$ divided by 2 and $D_{i-1}^{y}=$ block wise shift $D_{i-1}$, the number of shift will be total length of $D_{i-1}$ divided by 2 .

In Left Shifting Automatic Variable Key (LSAVK) [18] technique, the key is generated as per the following equation:

Subsequent key $K_{i}\left(\mathrm{i}^{\text {th }}\right.$ stage $)$ is generated by both sender and receiver as follows:

$$
\begin{gathered}
K_{1}^{\prime} \leftarrow K_{0} \oplus D_{0} \ldots \ldots \ldots \ldots \\
K_{1} \leftarrow \operatorname{CLS}\left(K_{1}^{\prime}\right) \ldots \ldots \ldots \\
K_{2}^{\prime} \leftarrow K_{1} \oplus D_{1} \ldots \ldots \ldots \\
K_{2} \leftarrow \operatorname{CLS}\left(K_{2}^{\prime}\right) \ldots \ldots \ldots \ldots .
\end{gathered}
$$

Where $K_{0}=$ Initial key and $D_{0}=$ First data set

Where CLS $\left(K_{i}^{s}\right)$ is circular left shift of $K_{0}^{s}$ by $n$ bits and continue this procedure until dataset $\neq \varnothing$.

We have compared the existing techniques namely, Computing \& Shifting Automatic Variable Key with key variation through noise burst bit, Alternating \& Shifting Automatic Variable Key with key variation through noise burst bit technique and Left shifting Automatic Variable Key with key variation through noise burst bit and found that these new techniques are more secure than Computing \& Shifting Automatic Variable Key technique without key variation noise burst bit, Alternating \& Shifting Automatic Variable Key technique without noise burst bit and Left shifting Automatic Variable Key without noise burst bit. In section 2, we have described our new schemes with examples. We have included our experimental results for performance analysis in section 3, section 4 summarizes comparison of the proposed schemes with existing ones followed by randomness verification in section 5. Finally, conclusion is given in section 6 .

\section{Proposed Scheme}

Here, we have proposed new techniques to generate new keys in Automatic Variable Key with key variation through noise burst bit (AVK with NB). The purpose is to yield 
new keys with noise burst to enhance security by generating more randomness between two successive keys.

The algorithm of the new technique is as follows:

- Initial key $\left(K_{1}\right)$ and noise burst will be exchanged between sender and receiver.

- $\quad$ Subsequent key $\left(K_{i}\right)$ is generated by both sender and receiver.

At first, noise burst will be read; the bits of noise burst will be read from left to right. If the bit of noise burst is 1 then new key will be generated as per Computing \& Shifting Automatic Variable Key (CSAVK) technique as per equation number (1).

No new key will be generated if bit of noise burst is 0 , the key will be as same as previously generated keys.

The procedure will be same as in case of Alternating \& Shifting Automatic Variable Key (ASAVK) technique. The bits of noise burst will be read from left to right and it is continued till the end of all bits in noise burst. If bit of noise burst is 1, key will be generated as per Alternating \& Shifting Automatic Variable Key (ASAVK) technique based on equation number (2-3). If noise burst is 0 , key will be as same as previously generated key.

In case of Left Shifting Automatic Variable Key (LSAVK), the procedure will be same. If bit of noise burst is 1 then new key will be generated as per equation number (47) otherwise no new key will be generated, the key will be same as previously generated key. This scheme is known as Key Variation with Noise Burst Bit.

Noise Burst is a true random number so if we pass these proposed techniques as mentioned with noise burst bit then we can say that these new techniques are more superior and be capable of producing more randomness between two of successive keys.

In these proposed schemes, we have compared the Computing \& Shifting Automatic Variable Key technique, Alternating \& Shifting Automatic Variable Key technique and Left Shifting Automatic Variable Key technique with key variation through Noise Burst Bit (KVNBB).

\subsection{Example1}

Let us assume, initial key $K_{1}=$ 01100111001111011110001010010000111100010010000010 100110100011001101111000101001000011110001000110001100111001101000001010 011100 and data $D_{1}=$ 010010100111010101101100011010010111010101110011001000000100001101100001 01100101011100110110000101110010001000000111010101110011 with noise burst $=111001010011$ 110111100010111101001111000100101010101001101010110111100101001000101111 01100110101011011111011100111100010010101010 then the new keys will be generated as follows:

$1^{\text {st }}$ bit of noise burst is 1 , so the next will be generated as per Computing \& Shifting Automatic Variable Key technique in equation number (1).

$K_{2}=$

101111000101010110010011010101010010111010000010101000000100111011111101

00101011000011110101111100000011100000100011101000110101

$2^{\text {nd }}$ bit of noise burst is 1 so the new key will be as follows:

$K_{3}=$

110110101010001010110000100100100110000100100110100001100101101001010111

00000000000011110101001010101000000011001011011000011100

$3^{\text {rd }}$ bit of noise burst is 1 so the new will be: 


$$
K_{4}=
$$

111001101011010111000100010000100010111110000111100101011110101011000100

01100100000001100111101100000110010001011100110100011001

$4^{\text {th }}$ bit of noise burst is 0 so the key will be as same as previous key

$$
K_{5}=
$$

111001101011010111000100010000100010111110000111100101011110101011000100 01100100000001100111101100000110010001011100110100011001 and procedure is continued until noise burst $\neq \varnothing$

\subsection{Example2}

Let us consider, the initial key $K_{1}=$ 0110011100111101111000101001000011110001001000 001010011010001100110111100010100100001111000100011000110011100110100000 101001110 and $D_{1}=$ 010010100111010101101100011010010111010101110011001000000100001101100001 01100101011100110110000101110010001000000111010101110011 with noise burst $=$ 11100101001

111011110001011110100111100010010101010100110101011011110010100100010111 101100110101011011111011100111100010010101010.

The new keys will be generated as follows:

$1^{\text {st }}$ bit of noise burst is 1 , hence the next will be generated as per Alternating \& Shifting Automatic Variable Key technique as per equation number (2-3).

$K_{2}$ 100101000101110001100011011110001111100110010101101000101101111100000110 01011000100100011111000110000011000000001101001111111111

$K_{3}$

111001000010100000001100000010111000000011100110110101101011101001100011 00111100101100011001000010100011011000111010000110000110

[Using the noise burst's $2^{\text {nd }}$ bit key $K_{3}$ has been generated]

$3^{\text {rd }}$ bit of noise burst is 1 so the new key will be generated as per Alternating \&Shifting Automatic Variable Key technique.

$K_{4}$

000011100001110011011000111111101000001100001011110010001111010111000100 01011111011011010111100111110011110010101111011011001101

$K_{5}$

011000000111001110101111110111101111000101101110101011101001000010101100 00110110000011100001000111010011101000111000010111101101

[Using noise burst's $4^{\text {th }}$ bit key $K_{5}$ has been generated] and procedure is continued until noise burst $\neq \emptyset$

\subsection{Example 3}

Let us assume, initial key $K_{1}=$ 01100111001111011110001010010000111100010010000010 100110100011001101111000101001000011110001000110001100111001101000001010 011100 and $D_{1}$ = 010010100111010101101100011010010111010101110011001000000100001101100001 01100101011100110110000101110010001000000111010101110011 with noise burst $=$ 111001010011 
110111100010111101001111000100101010101001101010110111100101001000101111 01100110101011011111011100111100010010101010

The new keys will be generated as per equation number (4-7) as follows:

$1^{\text {st }}$ bit of noise burst is 1 , hence the next will be generated as per Left Shifting Automatic Variable Key technique:

$K_{2}$ 100001000000111010101001010010001001011100110010011011010101101011011101 00110111011011010100001001101011111011010111000001001011

$2^{\text {nd }}$ bit of noise burst is 1 , so the keys will be as:

$K_{3}$

011101010101111010000101010000110111110010101010110001110001001000000100 10101001110110100111101011010110110001101011010101001000 and procedure is continued until dataset $\neq \varnothing$

2.4. Illustration of Computing \& Shifting Automatic Variable Key with Key Variation Noise Burst Bit (ASAVK with NB), Alternating \& Shifting Automatic Variable Key with Key Variation Noise Burst Bit (ASAVK with NB) and Left Shifting Automatic Variable Key with Key Variation Noise Burst Bit (LSAVK with NB)

Let us consider, sender sends initial key $K_{1}=$ 01100111001111011110001010010000111100 010010000010100110100011001101111000101001000011110001000110001100111001 101000001010011100 and data $D_{1}=$ 010010100111010101101100011010010111010101110011001000000100 00110110000101100101011100110110000101110010001000000111010101110011 with noise burst $=$ 111001010011110111100010111101001111000100101010101001101010110111100101 00100010111101100110101011011111011100111100010010101010

Then the new key will be generated as per Computing \& Shifting Automatic Variable Key technique in Equation number (1) when noise burst bit is 1, next key will be generated as:

1011110001010101100100110101010100101110100000101010000001001110111111 0100101011000011110101111100000011100000100011101000110101 and when noise burst is 0 then next key will be as same as previously generated key.

In case of Alternating \& Shifting Automatic Variable Key technique, the new key will be generated as per Equation (2-3) when bit of noise burst is 1

$K_{2}$

100101000101110001100011011110001111100110010101101000101101111100000110 01011000100100011111000110000011000000001101001111111111

$K_{3}$

111001000010100000001100000010111000000011100110110101101011101001100011 00111100101100011001000010100011011000111010000110000110 and when noise burst is 0 then the next key will be as same as previously generated keys.

Let us assume sender sends initial key $K_{1}=$ 01100111001111011110001010010000111100010 010000010100110100011001101111000101001000011110001000110001100111001101 000001010011100 and data $D_{1}=01001010011101010110110001101001011101010111001100100000010000110$ 110000101100101011100110110000101110010001000000111010101110011 with noise burst 
001010011110111100010111101001111000100101010101001101010110111100101001 00010111101100110101011011111011100111100010010101010

Then the new key will be generated as per Left Shifting Automatic Variable Key technique in Equation number (4-7) if the noise burst bit is 1 . So the key will be as:

$K_{2}$

100001000000111010101001010010001001011100110010011011010101101011011101

00110111011011010100001001101011111011010111000001001011

$2^{\text {nd }}$ bit of noise burst is 1 , so the keys will be as:

$K_{3}$

011101010101111010000101010000110111110010101010110001110001001000000100

10101001110110100111101011010110110001101011010101001000

\section{Performance Analysis}

For performance analysis of the proposed techniques, we have taken initial key as $K_{1}=$ 011001110011110111100010100100001111000100100000101001101000110011011110 00101001000011110001000110001100111001101000001010011100 and noise burst $=$ 11100101001111011110

001011110100111100010010101010100110101011011110010100100010111101100110

101011011111011100111100010010101010 with the following dataset

Dataset $=" J u l i u s$ Caesar used a cryptosystem in his wars, which is now referred to as Caesar cipher it is an additive cipher with the key set to three, each character in the plaintext is shifted three characters to create cipher text. A brute Force attack on a Hill cipher is extremely difficult because the key is an $m X$ m matrix, the key matrix in the Hill cipher needs to have a multiplicative inverse, multiplicative inverses are only defined for square matrices".

Using the same initial key and data set pairs with noise burst bit, we have calculated the randomness, average randomness and standard deviation and portrayed these from Figure 1 to 3 respectively. For analysis purpose, we have assumed randomness as a parameter which is a measure of amount of variation between successive keys. For example if $K_{i}=1010101110110010$ and $K_{i+1}=0011101011110011$. So the randomness between $K_{i}$ and $K_{i+1}$ is 5 . These results are found more productive and superior among these techniques with noise burst as per literature survey.

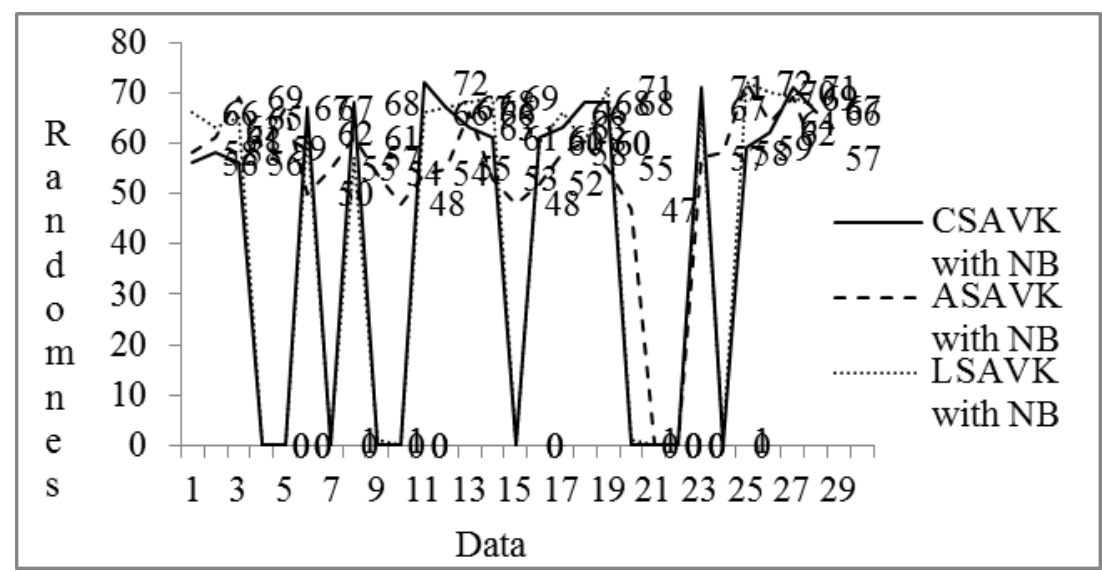

Figure 1. Randomness of keys of Computing \& Shifting Automatic Variable Key with Key Variation Noise Burst Bit (CSAVK with NB), Alternating \& Shifting Automatic Variable Key with Key Variation Noise Burst Bit (ASAVK with NB), Left Shifting Automatic Variable Key with Key Variation Noise Burst Bit (LSAVK with NB) 


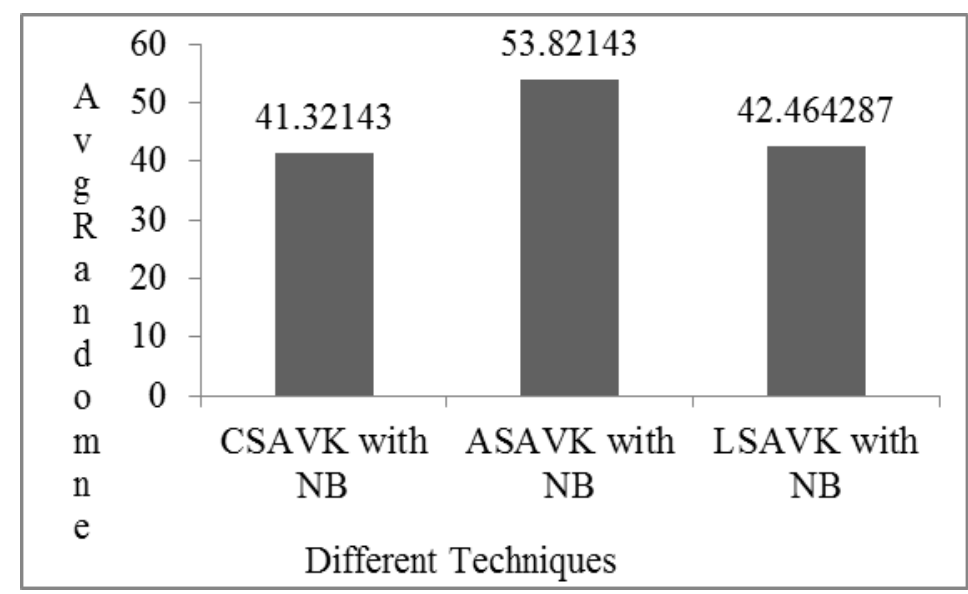

Figure 2. Comparison of Average Randomness of Computing \& Shifting Automatic Variable Key with Key Variation Noise Burst Bit (CSAVK with NB), Alternating \& Shifting Automatic Variable Key with Key Variation Noise Burst Bit (ASAVK with NB), Left Shifting Automatic Variable Key with Key Variation Noise Burst Bit (LSAVK with NB)

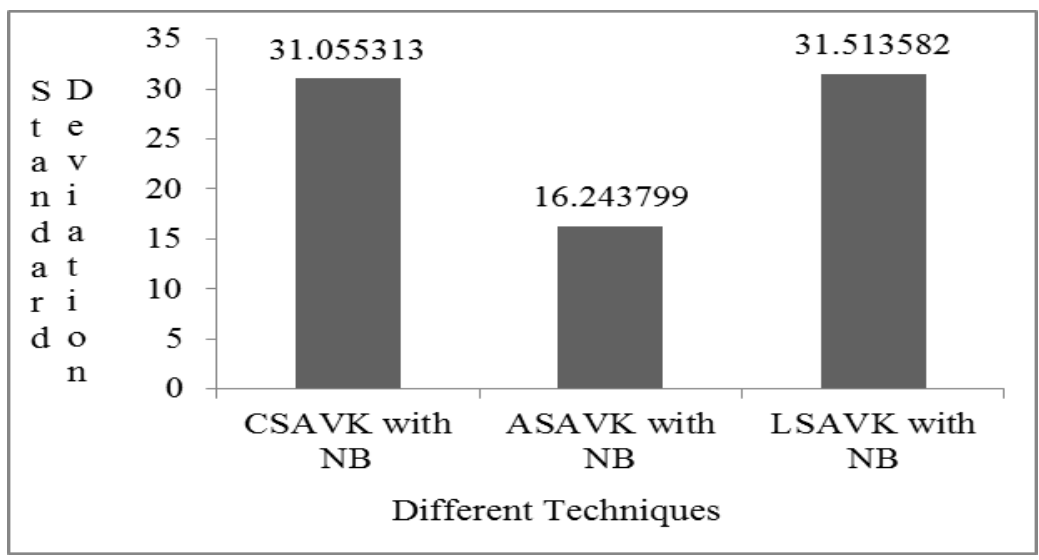

Figure 3. Comparison of Standard Deviation of Computing \& Shifting Automatic Variable Key with Key Variation Noise Burst Bit (CSAVK with NB), Alternating \& Shifting Automatic Variable Key with Key Variation Noise Burst Bit (ASAVK with NB), Left Shifting Automatic Variable Key with Key Variation Noise Burst Bit (LSAVK with NB)

\section{Comparison}

Here, we have proposed 3 new techniques and compared the results of average randomness and standard deviation of Computing \& Shifting Automatic Variable Key with key variation through noise burst bit, Alternating \& Shifting Automatic Variable Key with key variation through noise burst bit and Left Shifting Automatic Variable Key with key variation through noise burst bit with the existing ones namely, Computing \& Shifting Automatic Variable Key, Alternating \& Shifting Automatic Variable Key and Left Shifting Automatic Variable Key. The randomness and standard deviation have been calculated by executing those programs and results are portrayed in Figure 4 and 5 respectively. 


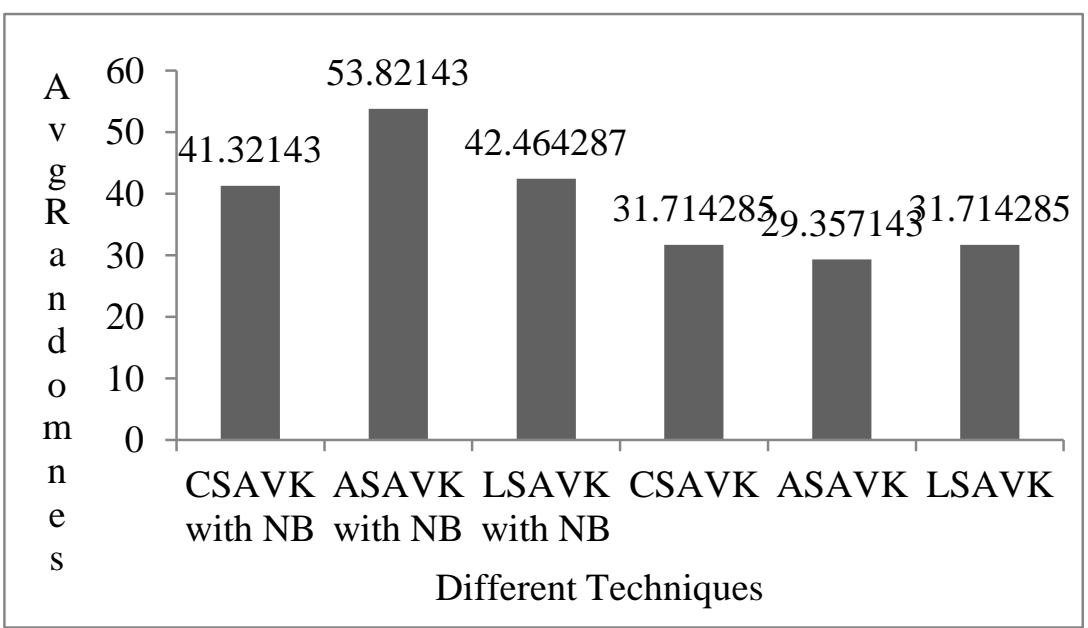

Figure 4. Comparison of Average Randomness of Computing \& Shifting Automatic Variable Key with Key Variation Noise Burst Bit (CSAVK with NB), Alternating \& Shifting Automatic Variable Key with Key Variation Noise Burst Bit (ASAVK with NB), Left Shifting Automatic Variable Key with Key Variation Noise Burst Bit (LSAVK with NB), Computing \& Shifting Automatic Variable Key (CSAVK), Alternating \& Shifting Automatic Variable Key (ASAVK), Left Shifting Automatic Variable Key (LSAVK)

From the graph of Figure 4 and 5, it is found that the superiority of one technique is higher than other technique (e.g. ASAVK with NB) and for other cases it is just opposite. From Figure 4, we have found that the Average Randomness of Alternating \& Shifting Automatic Variable Key with Noise Burst Bit technique is the highest than the other techniques.

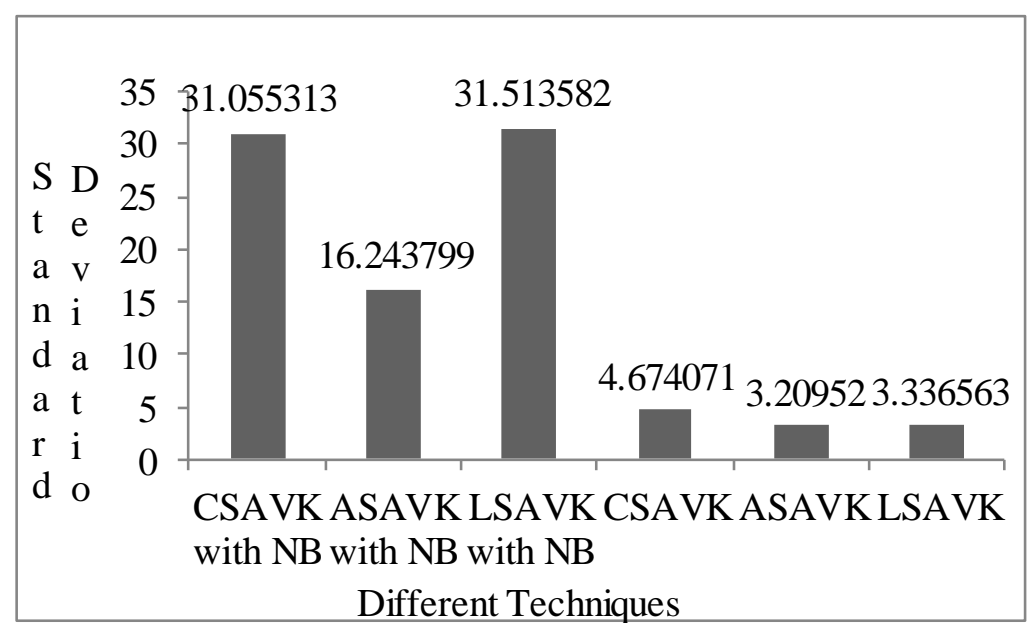

Figure 5. Comparison of Standard Deviation of Computing \& Shifting Automatic Variable Key with Key Variation Noise Burst Bit (CSAVK with NB), Alternating \& Shifting Automatic Variable Key with Key Variation Noise Burst Bit (ASAVK with NB), Left Shifting Automatic Variable Key with Key Variation Noise Burst Bit (LSAVK with NB), Computing \& Shifting Automatic Variable Key (CSAVK), Alternating \& Shifting Automatic Variable Key (ASAVK), Left Shifting Automatic Variable Key (LSAVK) 
The Standard Deviation of Left Shifting Automatic Variable Key with Noise Burst Bit is the highest as compared to other techniques as shown in Figure 5. Therefore, from the experiment, we have found that the superiority of different techniques may vary from one session to another session.

\section{Randomness Verification}

We have verified the randomness among the successive keys, generated by all the experiments defined in the previous section, with the help of NIST (National Institute of Standards \& Technology) test suite. The NIST test suite is a statistical package containing 15 different tests to determine whether a random key generator is suitable for a particular cryptographic application or not. We have examined the randomness of our successive keys based on the following 4 statistical tests only.

i) Frequency Test

ii) Block Frequency Test

iii) Cumulative Tests

iv) Runs Test

Each test is based on a calculated test statistic value, which is a function of data. If the test statistic value is $\mathrm{S}$ and the critical value is t, then the error probability is $\mathrm{P}\left(\mathrm{S}>\mathrm{t} \| \mathrm{H}_{0}\right.$ is true $)=\mathrm{P}$ (reject $\mathrm{H}_{0} \mid \mathrm{H}_{0}$ is true). Another type of error probability is $\mathrm{P}\left(\mathrm{S}<=\mathrm{t} \| \mathrm{H}_{0}\right.$ is false $)=\mathrm{P}\left(\right.$ accept $\mathrm{H}_{0} \mid \mathrm{H}_{0}$ is false $)$, here $H_{0}=$ null hypothesis. The test statistic is used to calculate a p-value that summarizes the strength of the evidence against the null hypothesis. For these tests each p-value is the probability that a perfect random number generator would have produced a sequence less random than the sequence that was tested, given the kind of non-randomness assesses by the test. If a p-value for a test is equal to 1 , then the sequence appears to have perfectly random. A p-value of zero indicates that the sequence appears to be completely non-random. If the p-value is $<0.01$ then it can be inferred that the sequence in non-random otherwise, the sequence is random. The p-values of our experiments have been summarized in Table 1:

Table 1. Statistical Test Results for Computing \& Shifting Automatic Variable Key with Key Variation Noise Burst Bit (CSAVK with NB), Alternating \& Shifting Automatic Variable Key with Key Variation Noise Burst Bit (ASAVK with NB) and Left shifting Automatic Variable Key with key Variation Noise Burst Bit (LSAVK with NB)

\begin{tabular}{|c|c|c|c|}
\hline $\begin{array}{l}\text { Proposed } \\
\text { Techniques }\end{array}$ & Test & P-Value & Remarks \\
\hline \multirow{5}{*}{$\begin{array}{lr}\text { Computing } & \text { \& } \\
\text { Shifting } & \\
\text { Automatic } & \\
\text { Variable } & \text { Key } \\
\text { with Noise } & \text { Burst } \\
\text { Bit } & \end{array}$} & Frequency Test & 0.213309 & Random \\
\hline & Block Frequency & 0.213309 & Random \\
\hline & $\begin{array}{l}\text { Cumulative } \\
\text { (Forward) }\end{array}$ & 0.534146 & Random \\
\hline & $\begin{array}{ll}\begin{array}{l}\text { Cumulative } \\
\text { (Backward) }\end{array} & \text { Sum } \\
\end{array}$ & 0.090936 & Random \\
\hline & Runs Test & 0.122325 & Random \\
\hline \multirow{4}{*}{$\begin{array}{lr}\text { Alternating } & \text { \& } \\
\text { Shifting } & \\
\text { Automatic } & \\
\text { Variable } & \text { Key } \\
\text { with } & \text { Key } \\
\text { Variation } & \text { with } \\
\end{array}$} & Frequency Test & 0.275709 & Random \\
\hline & Block Frequency & 0.275709 & Random \\
\hline & $\begin{array}{l}\text { Cumulative } \\
\text { (Forward) }\end{array}$ & 0.437274 & Random \\
\hline & $\begin{array}{l}\text { Cumulative } \\
\text { (Backward) }\end{array}$ & 0.739918 & Random \\
\hline
\end{tabular}




\begin{tabular}{|c|c|c|c|}
\hline Noise Burst Bit & Runs Test & 0.090936 & Random \\
\hline \multirow{5}{*}{$\begin{array}{lr}\text { Left Shifting } \\
\text { Automatic } \\
\text { Variable } \\
\text { with } \quad \text { Key } \\
\text { Variation rey } \\
\text { Noise Burst Bit } \\
\text { Nois }\end{array}$} & Frequency Test & 0.534146 & Random \\
\hline & Block Frequency Test & 0.534146 & Random \\
\hline & $\begin{array}{ll}\text { Cumulative } & \text { Sum } \\
\text { (Forward) } & \end{array}$ & 0.090936 & Random \\
\hline & $\begin{array}{l}\text { Cumulative } \\
\text { (Backward) }\end{array}$ & 0.739918 & Random \\
\hline & Runs Test & 0.350485 & Random \\
\hline
\end{tabular}

\section{Conclusion}

In our proposed schemes, we have calculated average randomness and standard deviation of the auto generated successive keys that can be applied in cryptography for security purpose in various applications. The generated keys of Computing \& Shifting Automatic Variable Key with key variation through noise burst bit, Alternating \& Shifting Automatic Variable Key with key variation through noise burst bit and Left Shifting Automatic Variable Key with key variation through noise burst bit are well established. After analysing the experiments, it is found that the superiority of Alternating \& Shifting Automatic Variable Key with key variation through noise burst bit for same dataset is much higher than other compared techniques when we use average randomness as parameter for comparison. But when we use standard deviation as a parameter, Left Shifting Automatic Variable Key with key variation through noise burst bit is much superior to other technique with noise burst. We have tested the generated key sequences in NIST (National Institute of Standards and Technology) statistical tool and we have seen that the key sequences are purely random. Hence, we can conclude that proposed techniques can be applied in cryptographic algorithms such as with RSA, AES, DES for attaining perfect secrecy.

\section{References}

[1] C. E. Shannon, "Communication Theory of Secrecy System", the Bell System Tech J, (1949).

[2] H. Eberle, "A High Speed DES Implementation for Network Application", Proceeding International Conference Cryptology, (1992).

[3] American National Standards Institute: Financial Institution Key Management, American Bankers Association, ANSI X9.17, (1985).

[4] A. Rukin, J. Soto, J. Nechvatal, M. Smid, E. Barker, S. Leigh, M. Levenson, M. Vangel, D. Banks, A. Heckert, J. Dray and S. Vo," A statistical test suite for random and pseudorandom number generators for cryptographic applications", NIST special publication, (2010).

[5] C. T. Bhunia, "New approach for selective AES towards tackling error propagation effect of AE", ASIAN Journal of Information Technology, vol. 5990, (2006), pp. 1017-1022.

[6] C. T. Bhunia, "Information Technology, Network and Internet", New age publication.

[7] C. T. Bhunia, "Implementation of AVK with chaos theory and studied thereof", J IUP Computer Science, vol. V, no. 4, (2011), pp. 22-32.

[8] B. K. Singh, S. Banerjee, M. P. Dutta and C. T. Bhunia, "Generation of Automatic Variable key to make secure communication", International conference on recent cognizance in wireless communication\& image processing-ICRCWIP, (2014).

[9] M. P. Dutta, S. Banerjee and C. T. Bhunia, "Two new schemes to generate automatic variable key to achieve the perfect security in insecure communication channel", Proceeding of the International Conference on Advanced Research in Computer Science Engineering \&Technology (ICARCSET), (2015); Eluru, India.

[10] R. S. Goswami, S. Chakraborty and C. T. Bhunia, "Various new methods of Implementing Automatic Variable Key", Preceding of the 2nd International Conference Advanced Computer science and Engineering, (2013).

[11] R. S. Goswami, S. Chakraborty, C. T. Bhunia and A. Bhunia, "New Approach towards generation of Automatic Variable Key to achieve perfect security", ITNG, IEEE Computer Society, CPS, (2013), pp. 489-491. 
[12] R. S. Goswami, S. Chakraborty and C. T. Bhunia, "Approach towards Optimum data transfer with various Automatic variable Key techniques to achieve perfect Security with analysis and comparison", International Journal of Computer Application, vol. 82, no. 1, (2013).

[13] R. S. Goswami, S. Chakraborty, C. T. Bhunia and A. Bhunia, "Generation of Automatic variable key under various approaches in cryptography system", Journal of Institute Engineering, India Ser. B, vol. 94, no. 4, pp. 215-220.

[14] C. T. Bhunia, R. S. Goswami and S. K. Chakraborty, "A new technique of AVK in achieving perfect security", 100th Indian Science Congress Association 3rd-7th, (2013).

[15] R. S. Goswami, S. Chakraborty, A. Bhunia and C. T. Bhunia, "New Techniques for generating of Automatic variable Key in achieving perfect security", Journal of Institute Engineering, India Ser. B, vol. 95, no. 3, (2014), pp. 197-201.

[16] R. S. Goswami, S. Banerjee, M. P. Dutta and C. T. Bhunia, "Absolute key variation technique of AVK in Cryptography",Preceding SIN of the 8th International Conference on security of Information and Networks (Sochi, Russia), (2015).

[17] R. S. Goswami, S. Chakraborty and C. T. Bhunia, "A study to examine the Superiority of CSAVK, AVK over conventional encryption with a single key", International Journal of Security and Its Applications, vol. 10, no. 2, (2016), pp. 279-286.

[18] M. Das, R. S. Goswami and C. T. Bhunia, "Implementation of New Method to Generate a Key in Automatic Variable Key for Perfect Security", International Journal of Security and Its Applications, vol. 10, no. 4, (2016), pp. 367-376.

[19] M. Das, P. Roy, R. S. Goswami and C. T. Bhunia, "Investigation a New Technique of Automatic Variable Key using Two Dimensional Matrix Approach to Achieve Perfect Security", communicated to Inderscience publisher, under processing.

[20] M. Das, R. S. Goswami, M. P. Dutta, S. K. Chakraborty and C. T. Bhunia, "New Approaches to Generate Variable Keys with Noise Burst Bit for Achieving Perfect Security in Modern Cryptosystem", Communicated to "International Journal of Information Security and Privacy", IGI-Global.

\section{Authors}

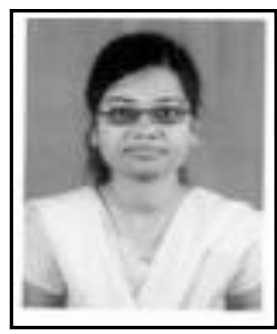

Moumita Das, she earned her M. Tech degree in Information Technology from Bengal Engineering \& Science University, Shibpur in 2013. She has teaching experience more than four years. She worked as a junior research fellow in the department of Electronics \& Communication Engineering at National Institute of Technology, Arunachal Pradesh, Govt. of India. She is currently pursuing $\mathrm{PhD}$ in the department of Computer science \& Engineering and working as a Guest Lecturer in the department of Computer science \& Engineering at National Institute of Technology, Arunachal Pradesh, Govt. of India.

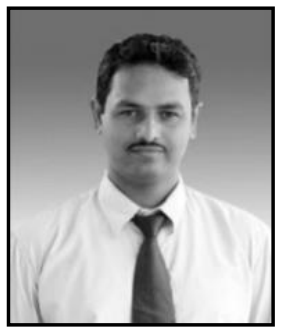

Rajat Subhra Goswami, he is working as an Assistant Professor in the department of Computer science \& Engineering in National Institute of Technology, Arunachal Pradesh, Govt of India. He is having more than 8 years of teaching experience. His research areas are cryptography and information security. He is the author of 20 peer reviewed publications. In 2013, he got international travel support grand from department of Science \& Technology to present one of his research papers at Los Angeles, USA. In 2013, he was awarded as best teacher for the academic year 2012-2013. He received his $\mathrm{PhD}$ degree from National Institute. 

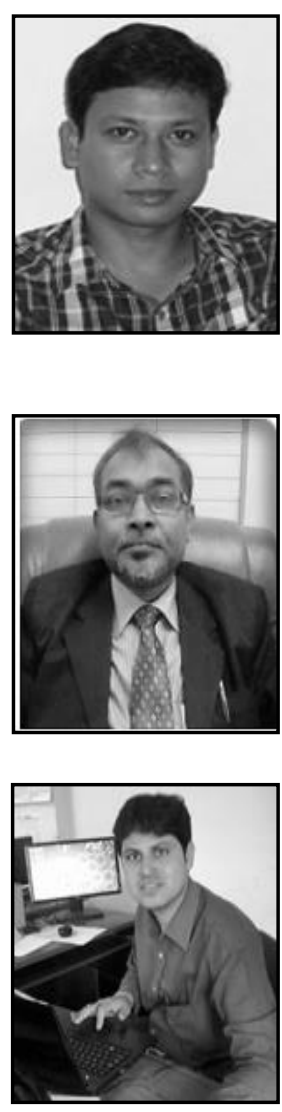

Manash P Dutta, he is a working as an Assistant Professor in the department of Computer science \& Engineering and pursuing Ph.D.in the department of Computer science \& Engineering in National Institute of Technology, Arunachal Pradesh, Govt. of India. He is having more than 6 years of teaching experience. His research activities are focused on cryptography and network security.

S.K. Chakraborty, he is working as an Assistant Professor and Head of the department in the department of Computer science \& Engineering in National Institute of Technology, Arunachal Pradesh, Govt. of India. He is having more than 8 years of teaching experience. His research areas are cryptography and information security. He received his $\mathrm{PhD}$ degree from National Institute of Technology, Arunachal Pradesh in 2015.

Chandan Tilak Bhunia earned his B. Tech in Radio Physics and Electronics in 1983 from Calcutta University then joined DVC of Govt. of India as telecommunication engineer. He got M. Tech degree in Radio Physics and Electronics in 1985 and then joined North Bengal University as a lecturer of computer science \& application in 1988, and became Assistant Professor of electronics \& communication engineering at the North Eastern Regional Institute of Science \& Technology (NERIST) of Govt. of India in 1990. He got his $\mathrm{PhD}$ in computer science \&engineering from the Jadavpur University. He became full Professor in 1997 at NERIST where he was HOD of ECE \& CSE for about 6yrs and Dean (Academics/Post Graduate Studies) for about 1.5 years. He then switched to private engineering colleges from 1999 to 2003 as HOD, Deputy Director and Director. Lastly, he was a full Professor of computer science \& engineering of the Indian School of Mines (Deemed University) of Govt of India. He was a senior Professor and Dy Director (Acad) of Haldia Institute of Technology. He has extensively visited foreign countries, namely China, Italy, Singapore, UK and Bangladesh on several assignments including BOYSCAST Fellowship and ICTP senior associate ship. He has published around 200 research papers and technical articles/reports in national/international journals/magazines/seminars. He is the author of the books a) "Introduction to Knowledge Management" published by Everest Publishing House, Pune in 2003 and b) "Information Technology, Network and Internet", published by the New Age International Publishers, New Delhi. He is fellow of IETE and the IE (I) and a senior member of IEEE and CSI. He is currently as a Director of National Institute of Technology, Arunachal Pradesh. 